\title{
TRACE PALEY-WIENER THEOREM IN THE TWISTED CASE
}

\author{
J. D. ROGAWSKI
}

\begin{abstract}
A version of the trace Paley-Wiener theorem for a reductive $p$ adic group in the context of twisted harmonic analysis with respect to an outer automorphism is proved.
\end{abstract}

Let $\mathbf{G}$ be a connected reductive algebraic group over a $p$-adic field $F$ (of characteristic 0 ) and let $G=\mathbf{G}(F)$. Let $P_{0}$ be a minimal parablic subgroup of $G$. Let $\varepsilon$ be an outer automorphism of $\mathbf{G}$ of finite order $r$ of $G$ and suppose that $\varepsilon$ fixes $P_{0}$. Elements $x, y \in G$ are called $\varepsilon$-conjugate if $y=g^{-1} x \varepsilon(g)$ for some $g \in G$. A distribution on $G$ which is invariant under twisted conjugacy is called $\varepsilon$-invariant. The objects of study in twisted harmonic analysis are $\varepsilon$-invariant distributions. The main examples are provided by twisted orbital integrals (invariant integrals over twisted conjugacy classes) and by twisted characters, which are obtained as follows. Let $\pi$ be an irreducible admissible representation of $G$ and let $\varepsilon(\pi)$ denote the representation of $G$ through which $g \in G$ acts by $\pi\left(\varepsilon^{-1}(g)\right)$. If $\pi$ is equivalent to $\varepsilon(\pi)$, then there exists an operator $\pi(\varepsilon)$, unique up to $r$ th roots of unity, such that $\pi(\varepsilon) \pi(g) \pi(\varepsilon)^{-1}=\pi(\varepsilon(g))$ and $\pi(\varepsilon)^{r}=1$. In other words, $\pi$ extends to the semidirect product $G^{*}=G \rtimes\langle\varepsilon\rangle$. The twisted character of $\pi$ (which depends on the choice of $\pi(\varepsilon))$ is the distribution $f \rightarrow \operatorname{Tr}(\pi(f) \pi(\varepsilon))$ on the space $\mathcal{H}(G)$ of compactly supported locally constant functions on $G$.

In applications of the trace formula to global problems in the theory of automorphic representations, it is often necessary to use results on the representation theory and harmonic analysis of reductive groups over $p$-adic fields. Because of the role played by twisted local harmonic analysis in applications of the twisted trace formula (e.g., $[\mathbf{1}, \mathbf{1 0}])$, it is important to have available extensions to the twisted case of results known for ordinary harmonic analysis.

Many results in harmonic analysis extend to the twisted case. For example, by [7], Harish-Chandra's theorem on the local integrability of characters holds for twisted characters. The purpose of this paper is to prove a twisted version (Proposition 11.2) of the trace Paley-Wiener theorem for $p$-adic groups of Bernstein, Deligne, and Kazhdan, given in [3]. The twisted Paley-Wiener theorem gives a characterization of functions of the form $\pi \rightarrow \operatorname{Tr}(\pi(f) \pi(\varepsilon))$ for some $f \in \mathcal{H}(G)$.

In $\S \S 1-6$, the appropriate setting for the twisted theorem is developed. In $\S \S 7-$ 10, results needed for the proof of Proposition 11.2 are proved. Proposition 7.4 is a twisted version of Casselman's theorem on characters. The proof of Proposition 11.2 follows the plan of the argument given in [3], with modifications for the twisted

Received by the editors December 12, 1986 and, in revised form, June 11, 1987.

1980 Mathematics Subject Classification (1985 Revision). Primary 22E50.

The author was partially supported by grants from the National Science Foundation and the Sloan Foundation. 
case introduced as needed. In particular, in $\S 10$, a finiteness theorem for $\varepsilon$-discrete representations is proved. In the ordinary case, a representation whose character cannot be written as a linear combination of characters of representations induced from proper parabolic subgroups is called discrete (or elliptic, because, by a theorem of D. Kazhdan, $\pi$ is discrete if and only if its character is not identically zero on the elliptic regular set). The twisted analogue " $\varepsilon$-discrete" is defined in $\S 9$. The end of a proof is denoted by a symbol.

1. Let $P_{0}=M_{0} N_{0}$ be a Levi decomposition of the minimal parabolic subgroup $P_{0}$ of $G$. We are assuming that $\varepsilon\left(P_{0}\right)=P_{0}$ and hence $\varepsilon\left(M_{0}\right)=n^{-1} M n$ for some $n \in N$ such that $n \varepsilon(n) \cdots \varepsilon^{r-1}(n)=1$. The group $N$ is an extension of additive groups and it is easily seen that $n=n_{1}^{-1} \varepsilon\left(n_{1}\right)$ for some $n_{1} \in N$. Replacing $M_{0}$ by a conjugate if necessary, we may assume that $\varepsilon\left(M_{0}\right)=M_{0}$. Let $A_{0}$ be the maximal split torus in the center of $M_{0}$.

A subgroup $M$ of $G$ will be called a Levi subgroup if $M \supset M_{0}$ and $M$ is a Levi factor of a parabolic subgroup. A Levi factor $M$ will be called standard if $M P_{0}$ is a parabolic subgroup and $M$ is a Levi factor of $M P_{0}$. A parabolic subgroup of the form $M P_{0}$ with $M$ standard will be called a standard parabolic subgroup. Let $\mathcal{L}(G)$ be the set of standard Levi subgroups. For $M \in \mathcal{L}(G)$, we define standard Levi subgroups of $M$ with respect to $M_{0}$ and the minimal parabolic subgroup $M \cap P_{0}$ of $M$.

Let $N_{M}\left(A_{0}\right)$ be the normalizer of $A_{0}$ in $M$ and let $W_{M}=N_{M}\left(A_{0}\right) / M_{0}$ be the Weyl group of $M$. Let $A_{M}$ denote the split component of $M$, and let $Z_{M}$ be the center of $M$. For $H$ an algebraic group over $F$, let $X(H)$ be the group of rational characters of $H$. Set $A(M)=\operatorname{Hom}\left(X\left(A_{M}\right), \mathbf{Z}\right) \otimes \mathbf{C}$.

If $M$ is an $\varepsilon$-invariant Levi subgroup, set $M^{*}=M \rtimes\langle\varepsilon\rangle$. The set of $\varepsilon$-invariant standard Levi subgroups will be denoted by $\mathcal{L}(G)^{\varepsilon}$. Note that if $M \in \mathcal{L}(G)^{\varepsilon}$, then the parabolic subgroup $M P_{0}$ is $\varepsilon$-invariant.

Let $M \in \mathcal{L}(G)$. A smooth representation $(\pi, V)$ of $M$ will be called an $M$ module. We will call $\pi$ square-integrable if $\pi$ is unitary and the matrix coefficients of $\pi$ are square-integrable modulo $Z_{M}$. Similarly, we use the term tempered to mean tempered and unitary. Suppose that $M \in \mathcal{L}(G)^{\varepsilon}$. A representation of $M^{*}$ will be called an $M^{*}$-module (resp. admissible, tempered, etc.) if its restriction to $M$ is smooth (resp. admissible, tempered, etc.). An $M^{*}$-module is thus an $M$-module $(\pi, V)$ together with an operator $\pi(\varepsilon)$ of order $r$ such that $\pi(\varepsilon(m))=$ $\pi(\varepsilon) \pi(m) \pi(\varepsilon)^{-1}$. Let $\operatorname{Irr}(M)$ (resp. $\operatorname{Irr}\left(M^{*}\right)$ ) be the set of equivalence classes of irreducible $M$-modules (resp. $M^{*}$-modules).

2. If $\varsigma$ is an $r$ th root of unit, and $(\pi, V)$ is an $M^{*}$-module, let $\left(\pi_{\varsigma}, V_{\varsigma}\right)$ be the $M^{*}$-module such that $V_{\varsigma}=V, \pi\left|M=\pi_{\varsigma}\right| M$, and $\pi_{\varsigma}(\varepsilon)=\varsigma \pi(\varepsilon)$. Let $R_{0}\left(M^{*}\right)$ be the Grothendieck group of $M^{*}$-modules of finite length. Let $R\left(M^{*}\right)$ be the quotient of $R_{0}\left(M^{*}\right) \otimes \mathrm{C}$ modulo the relations

$$
\pi_{\varsigma}-\varsigma \pi=0
$$

for all $M^{*}$-modules $\pi$ and all $r$ th roots of unity $\zeta$.

If $(\pi, V)$ is an $M$-module, let $(\varepsilon(\pi), \varepsilon(V))$ denote the module with the same underlying space $V$ on which $M$ acts by $m \rightarrow \pi\left(\varepsilon^{-1}(m)\right)$. 
LEMMA 2.1. If $(\pi, V) \in \operatorname{Irr}\left(M^{*}\right)$, then there is an irreducible $M$-submodule $\left(\pi_{0}, V_{0}\right)$ of $\pi$ and an integer $t$ dividing $r$ such that:

(a) $\pi_{0}, \varepsilon\left(\pi_{0}\right), \ldots, \varepsilon^{t-1}\left(\pi_{0}\right)$ are inequivalent and $\varepsilon^{t}\left(\pi_{0}\right)$ is equivalent to $\pi_{0}$.

(b) $\pi \mid M$ is isomorphic to $\pi_{0} \oplus \varepsilon\left(\pi_{0}\right) \oplus \cdots \oplus \varepsilon^{t-1}\left(\pi_{0}\right)$.

(c) As an $M^{*}$-module, $V$ is isomorphic to $V_{0} \oplus \varepsilon\left(V_{0}\right) \oplus \cdots \oplus \varepsilon^{t-1}\left(V_{0}\right)$, where $\varepsilon$ acts by $\left(v_{0}, v_{1}, \ldots, v_{t-1}\right) \rightarrow\left(T v_{t-1}, v_{1}, \ldots, v_{t-2}\right)$ for some linear operator $T$ on $V_{0}$ such that $T \pi_{0}(m) T^{-1}=\pi_{0}\left(\varepsilon^{t}(m)\right)$ for $m \in M$ and $T^{r / t}=1$.

PROOF. The restriction of $\pi$ to $M$ is of finite length and hence contains an irreducible $M$-submodule $V^{\prime}$. Let $V_{0}$ be the sum of all irreducible submodules of $V$ which are isomorphic to $V^{\prime}$. Let $t$ be the least integer such that $\varepsilon^{t}\left(V^{\prime}\right)$ is isomorphic to $V^{\prime}$. The operator $\pi(\varepsilon)^{t}$ preserves $V_{0}$. The sum $V_{0}+\pi(\varepsilon)\left(V_{0}\right)+\cdots+\pi(\varepsilon)^{t-1}\left(V_{0}\right)$ is direct and $M^{*}$-stable, and hence is all of $V$. Let $T$ be the restriction of $\pi(\varepsilon)^{t}$ to $V_{0}$. The map

$$
\left(v_{0}, v_{1}, \ldots, v_{t-1}\right) \rightarrow v_{0}+\pi(\varepsilon) v_{1}+\cdots+\pi(\varepsilon)^{t-1} v_{t-1}
$$

defines an $M$-isomorphism $V_{0} \oplus \varepsilon\left(V_{0}\right) \oplus \cdots \oplus \varepsilon^{t-1}\left(V_{0}\right) \rightarrow V$ which also commutes with the action of $\pi(\varepsilon)$, defined on the source as in the statement of the lemma. The irreducibility of $V_{0}$ follows from that of $V$.

Let $\operatorname{Irr}\left(M^{*}\right)^{0}$ be the set of $\pi \in \operatorname{Irr}\left(M^{*}\right)$ such that $t>1$, where $t$ is defined as in the previous lemma. Let $\operatorname{Irr}(M)^{\varepsilon}$ be the set of $\pi \in \operatorname{Irr}(M)$ such that $\varepsilon(\pi)=\pi$. For each $\pi \in \operatorname{Irr}(M)^{\varepsilon}$, we choose, once and for all, an extension of $\pi$ to $M^{*}$ and regard $\operatorname{Irr}(M)^{\varepsilon}$ as a subset of $\operatorname{Irr}\left(M^{*}\right)$. Then $R\left(M^{*}\right)$ is generated by the union of $\operatorname{Irr}(M)^{\varepsilon}$ and $\operatorname{Irr}\left(M^{*}\right)^{0}$. Let $R\left(M^{*}\right)^{0}$ be the subgroup of $R\left(M^{*}\right)$ generated by $\operatorname{Irr}\left(M^{*}\right)^{0}$.

The group $M^{*} / M$ is cyclic of order $r$. If $t$ divides $r$, let $\Sigma_{t}$ be the permutation representation of $M^{*} / M$ of dimension $t$.

LEMMA 2.2. Let $\pi_{0}$ be an $M^{*}$-module of finite length and set $\pi=\pi_{0} \otimes \Sigma_{t}$, where $t$ divides $r$. Then the image of $\pi$ in $R\left(M^{*}\right)$ lies in $R\left(M^{*}\right)^{0}$.

ProOF. Let $\mu$ be a primitive $t$ th root of unity. Then $\pi$ is isomorphic to the direct sum

$$
\left(\pi_{0}\right) \oplus\left(\pi_{0}\right)_{\mu} \oplus \cdots \oplus\left(\pi_{0}\right)_{\mu^{t-1}}
$$

and thus maps to zero in $R\left(M^{*}\right)$.

COROLLARY 2.3. Let $(\pi, V)$ be an $M^{*}$-module of finite length with the following property: there is an $M$-stable subspace $V_{0}$ of $V$ and an integer $t>1$ dividing $r$ such that $V=V_{0} \oplus \pi(\varepsilon)\left(V_{0}\right) \oplus \cdots \oplus \pi(\varepsilon)^{t-1}\left(V_{0}\right)$ and $\pi(\varepsilon)^{t} V_{0}=V_{0}$. Then the image of $(\pi, V)$ in $R\left(M^{*}\right)$ lies in $R\left(M^{*}\right)^{0}$.

ProOF. Let $W$ be an irreducible $M$-submodule of $V_{0}$. Let $s$ be the least integer such that $\varepsilon^{s}(W)$ is equivalent to $W$. Let $U=W \oplus \pi(\varepsilon)(W) \oplus \cdots \oplus \pi(\varepsilon)^{s-1}(W)$ and let $U_{j+1}=\pi(\varepsilon)^{j s} U$. As an $M$-module, $U$ is a direct sum of distinct irreducible modules and hence $U_{i} \cap U_{j} \neq\{0\}$ if and only if $\pi(\varepsilon)^{i s} W=\pi(\varepsilon)^{j s} W$, that is, if and only if $U_{i}=U_{j}$. We may choose $k$ such that $U_{1}, \ldots, U_{k}$ are distinct and $U_{k+1}=U_{1}$. The subspace $V^{\prime}=U_{1} \oplus \cdots \oplus U_{k}$ is $M^{*}$-stable. If $s>1, V^{\prime}$ is a direct sum of elements of $\operatorname{Irr}\left(M^{*}\right)^{0}$. If $s=1$, extend $U$ to an $M^{*}$-module $\pi_{0}$ such that $\pi_{0}(\varepsilon)^{k}$ is the restriction of $\pi(\varepsilon)^{k}$ to $U$. Then $V^{\prime}$ is isomorphic to $\pi_{0} \otimes \Sigma_{k}$. The corollary follows from the lemma and induction on the length of $\pi$. 
3. Let $L, M \in \mathcal{L}(G)$ and suppose that $L \subset M$. Let $i_{M L}$ be the standard induction functor from $L$-modules to $M$-modules. If $(\rho, V)$ is an $L$-module, then $i_{M L}(\rho)$ denotes the module unitarily induced from the parabolic subgroup $L\left(M \cap N_{0}\right)$ of $M$. The space of $\pi$ consists of functions $\varphi: M \rightarrow V$ such that $\varphi(m n g)=\delta_{N}(m)^{1 / 2} \rho(m) \varphi(g)$ for $m \in L, n \in N, g \in M$, where $N$ is the unipotent radical of $L\left(M \cap N_{0}\right)$ and $\delta_{N}$ is the modulus character of $L$ with respect to $N$.

If $L, M \in \mathcal{L}(G)^{\varepsilon}$ is a subgroup of $M$ and if $(\rho, V)$ is an $L^{*}$-module, then $\pi=$ $i_{M L}(\rho \mid L)$ has a natural $M^{*}$-module structure. The operator $\pi(\varepsilon)$ is defined by $(\pi(\varepsilon) \varphi)(g)=\rho(\varepsilon) \varphi\left(\varepsilon^{-1}(g)\right)$. This $M^{*}$-module will also be denoted by $i_{M L}(\rho)$.

Similarly, $r_{L M}$ will denote the standard Jacquet functor. If $(\pi, V)$ is an $M$ module, the space of $r_{L M}(\pi)$ is $V_{N}=V / V(N)$ where $V(N)$ is the span of vectors of the form $\pi(n) v-v$ for $n \in N$ and $v \in V$. An element $m \in L$ acts by $v+V(N) \rightarrow$ $\delta_{N}(m)^{-1 / 2} \pi(m) v+V(N)$. If $L, M \in \mathcal{L}(G)^{\varepsilon}$ and if $\pi$ is an $M^{*}$-module, then $V(N)$ is $\varepsilon$-stable since $\varepsilon(N)=N$ and thus $\varepsilon$ acts on $V_{N}$. This makes $r_{L M}(\pi)$ into an $L^{*}$-module which will also be denoted by $r_{L M}(\pi)$.

We obtain morphisms $i_{M L}: R_{0}\left(L^{*}\right) \rightarrow R_{0}\left(M^{*}\right)$ and $r_{L M}: R_{0}\left(M^{*}\right) \rightarrow R_{0}\left(L^{*}\right)$. As in the nontwisted case, $r_{L M}$ is left adjoint to $i_{M L}$. If $(\pi, V)$ and $(\rho, W)$ are $M^{*}$ and $L^{*}$-modules, respectively, and $\varphi: V_{N} \rightarrow W$ is a map of $L^{*}$-modules, we obtain the corresponding $M^{*}$-module map $\varphi^{\prime}: V \rightarrow i_{M L}(W)$ by sending $v \in V$ to the function $\varphi_{v}^{\prime}: m \rightarrow \varphi(\pi(m) v)$. Now $\varepsilon(v)$ is mapped to the function

$$
\varphi_{\varepsilon(v)}^{\prime}(m)=\varphi(\pi(m) \varepsilon(v))=\varphi\left(\pi(\varepsilon) \pi\left(\varepsilon^{-1}(m)\right) v\right)=\rho(\varepsilon) \varphi\left(\pi\left(\varepsilon^{-1}(m)\right) v\right)
$$

and this is equal to $i_{M L}(W)(\varepsilon)\left(\varphi_{v}^{\prime}\right)(m)$. This defines a map from $\operatorname{Hom}_{L^{*}}\left(V_{N}, W\right)$ to $\operatorname{Hom}_{M^{*}}\left(V, i_{M L}(W)\right)$ which is easily seen to be a bijection. The functors $i_{M L}$ and $r_{M L}$ induce morphisms on $R\left(L^{*}\right)$ and $R\left(M^{*}\right)$. However, $i_{M L}$ is not necessarily injective on $R\left(L^{*}\right)$.

4. An element of $\operatorname{Hom}_{\mathbf{C}}\left(R\left(M^{*}\right), \mathbf{C}\right)$ is determined by its values on the elements of $\operatorname{Irr}\left(M^{*}\right)$. Let $R\left(M^{*}\right)^{\prime}$ be the subspace of elements of $\operatorname{Hom}_{\mathrm{C}}\left(R\left(M^{*}\right), \mathrm{C}\right)$ which vanish on $\operatorname{Irr}\left(M^{*}\right)^{0}$. An element of $R\left(M^{*}\right)^{\prime}$ is determined by its values on $\operatorname{Irr}(M)^{\varepsilon}$.

LEMMA 4.1. If $F \in R\left(M^{*}\right)^{\prime}$, then the function $\rho \rightarrow F\left(i_{M L}(\rho)\right)$ lies in $R\left(L^{*}\right)^{\prime}$. If $F \in R\left(L^{*}\right)^{\prime}$, then the function $\pi \rightarrow F\left(r_{L M}(\pi)\right)$ lies in $R\left(M^{*}\right)^{\prime}$.

PROOF. For the first assertion, observe that if $\rho \in \operatorname{Irr}\left(M^{*}\right)^{0}$, then $i_{M L}(\rho)$ satisfies the hypothesis of Corollary 2.3. Hence $i_{M L}(\rho) \in R\left(M^{*}\right)^{0}$ and $F\left(i_{M L}(\rho)\right)=0$. It is clear that $r_{L M}$ maps $\operatorname{Irr}\left(M^{*}\right)^{0}$ to $R\left(L^{*}\right)^{0}$ and the second assertion follows.

This lemma shows that $i_{M L}$ and $r_{L M}$ define maps $i_{M L}^{*}: R\left(L^{*}\right)^{\prime} \rightarrow R\left(M^{*}\right)^{\prime}$ and $r_{L M}^{*}: R\left(M^{*}\right)^{\prime} \rightarrow R\left(L^{*}\right)^{\prime}$.

5. For $M \in \mathcal{L}(G)$, let $\mathcal{H}(M)$ be the Hecke algebra of locally constant functions on $M$ of compact support. For $K$ an open compact subgroup of $M$, let $\mathcal{H}_{K}(M)$ be the subalgebra of $\mathcal{H}(M)$ consisting of the bi- $K$-invariant elements. Every open compact subgroup contains an $\varepsilon$-invariant one and hence $H(M)$ is the union of the $\mathcal{H}_{K}(M)$ for $K \varepsilon$-invariant.

Fix a choice of Haar measure $d m$ on $M$. For $f \in \mathcal{H}(M)$ define

$$
\chi_{f}(\pi)=\operatorname{Trace}(\pi(f) \pi(\varepsilon)),
$$

where $\pi(f)=\int f(m) \pi(m) d m$. It is clear that $\chi_{f}(\pi)=0$ if $\pi \in \operatorname{Irr}\left(M^{*}\right)^{0}$, and hence $\chi_{f} \in R\left(M^{*}\right)^{\prime}$. 
Let $F_{\mathrm{tr}}(M)$ be the subspace of $R\left(M^{*}\right)^{\prime}$ of functions of the form $\chi_{f}$. Elements of $F_{\mathrm{tr}}(M)$ will be called trace functions. As in the nontwisted case, the Paley-Wiener theorem gives a characterization of $F_{\mathrm{tr}}(G)$ in $R\left(G^{*}\right)^{\prime}$.

6. Let $Z(G)$ denote the Bernstein center of $G[2]$. Recall that an element $z \in$ $Z(G)$ may be defined as a collection $\left\{z(\pi) \in \operatorname{End}_{M}(\pi)\right\}$, where $\pi$ ranges over all $M$-modules, such that the assignment $\pi \rightarrow z(\pi)$ commutes with all maps of $G$ modules. In particular, $z$ defines an endomorphism of $\mathcal{H}(G)$. If $\pi \in \operatorname{Irr}(G)$, then $z(\pi)$ is a scalar.

A cuspidal pair is a pair $(M, \rho)$ where $M \in \mathcal{L}(G)$ and $\rho$ is a supercuspidal representation of $M$. The set of all cuspidal pairs up to conjugation by $W$ will be denoted by $\Theta(G)$. By definition, the image of the map $X(M) \rightarrow \Theta(G)$ defined by $\psi \rightarrow(M, \rho \psi)$ for a cuspidal pair $(M, \rho)$ is called a connected component of $\Theta(G)$. The image of $X(M)$ in $\Theta(G)$ is given the structure of complex affine algebraic variety as a quotient of $X(M)$. According to Bernstein's theorem [2], for $(M, \rho)$ cuspidal and $\psi \in X(M)$, an element $z \in Z(G)$ acts by a scalar on $i_{G M}(\chi \rho)$ whose dependence on $\chi$ is polynomial. This defines an isomorphism of $Z(G)$ with the algebra of regular functions on $\Theta(G)$. For $M \in \mathcal{L}(G), \Theta(M)$ and $Z(M)$ are defined similarly.

If $L, M \in \mathcal{L}(G)$ and $L \subset M$, then a homomorphism $i_{M L}^{*}: Z(M) \rightarrow Z(L)$ is defined in [3] as the adjoint to the natural map $\Theta(L) \rightarrow \Theta(M)$. Since a cuspidal pair in $\Theta(L)$ may be conjugate under $W_{M}$ but not under $W_{L}$, the map is not an inclusion. However, $Z(L)$ becomes a finitely-generated $Z(M)$-module via $i_{M L}^{*}$.

Assume that $M \in \mathcal{L}(G)^{\varepsilon}$. Then the vector space $R\left(M^{*}\right)^{\prime}$ has a natural $Z(M)$ module structure. For $z \in Z(M)$ and $F \in R\left(M^{*}\right)^{\prime}, z F$ is the function whose value is $z(\pi) F(\pi)$ at $\pi \in \operatorname{Irr}(M)^{\varepsilon}$. If $f \in \mathcal{H}(M)$ and $z \in Z(M)$, then

$$
\chi_{z f}(\pi)=\operatorname{Trace}(\pi(z f) \pi(\varepsilon))=z(\pi) \operatorname{Trace}(\pi(f) \pi(\varepsilon))
$$

and hence $z \chi_{f}=\chi_{z f}$. In particular, $F_{\mathrm{tr}}(M)$ is a $Z(M)$-submodule of $R\left(M^{*}\right)^{\prime}$. If $L \in \mathcal{L}(G)^{\varepsilon}$ and $L \subset M$, then $R\left(L^{*}\right)^{\prime}$ has the structure of a $Z(M)$-module via the homomorphism $Z(M) \rightarrow Z(L)$.

Let $z \in Z(M)$. For each $L$-module $\rho, i_{M L}^{*}(z)$ defines an endomorphism of $\rho$ and induces an endomorphism $i_{M L}\left(i_{M L}^{*}(z)\right)$ on $i_{M L}(\rho)$ by functoriality. By Proposition 2.4 of [3], $i_{M L}\left(i_{M L}^{*}(z)\right)=z$ on $i_{M L}(\rho)$. Similarly, for each $M$-module $\pi$, $z$ induces an endomorphism $r_{L M}(z)$ on $r_{L M}(\pi)$ and Proposition 2.4 of [3] asserts that $r_{L M}(z)=i_{M L}^{*}(z)$ on $r_{L M}(\pi)$. This implies that the morphisms

$$
i_{M L}^{*}: R\left(M^{*}\right)^{\prime} \rightarrow R\left(L^{*}\right)^{\prime}, \quad r_{L M}^{*}: R\left(L^{*}\right)^{\prime} \rightarrow R\left(M^{*}\right)^{\prime}
$$

are maps of $Z(M)$-modules.

7. The following propositions is the twisted analogue of Proposition 3.2 of [3].

Proposition 7.1. For all $M \in \mathcal{L}(G)^{\varepsilon}, r_{G M}^{*}\left(F_{\mathrm{tr}}(M)\right) \subset F_{\mathrm{tr}}(G)$.

Let $M \in \mathcal{L}(G)^{\varepsilon}$. Let $P=M P_{0}$ and let $N$ be the unipotent radical of $P$. Let $P^{-}=M N^{-}$be the opposite parabolic subgroup. An element $m \in M$ is said to be strictly contracting on $N$ if for any two open compact subsets $U_{1}, U_{2}$ of $N$, there exists a positive integer $k$ such that $\operatorname{Ad}\left(m^{k}\right) U_{1} \subset U_{2}$. Let $M^{+}$be the set of elements $m \in M$ such that $\operatorname{Ad}(m)$ is strictly contracting on $N$. 
Let $K$ denote a small ( $\varepsilon$-invariant) open compact subgroup of $G$ which is "good" in the sense that the following decomposition holds:

$$
K=\left(K \cap N^{-}\right)(K \cap M)(K \cap N) .
$$

The decomposition of an element $g \in K$ as $n^{-} m n$ is unique and hence the intersection of good open compact subgroups is again good. Arbitrarily small good open compact subgroups $K$ are known to exist, and if $K$ is good, then $\bigcap \varepsilon^{i}(K)$ is good and $\varepsilon$-invariant. Hence arbitrarily small good $\varepsilon$-invariant open compact subgroups $K$ exist.

Set $K^{0}=K \cap M, K^{+}=K \cap N^{-}, K^{-}=K \cap N$. It will suffice to prove that $r_{G M}^{*}\left(\chi_{f}\right) \in F_{\mathrm{tr}}(G)$ for all $f \in \mathcal{H}_{K^{\circ}}(M)$ for $K \varepsilon$-invariant and good.

For a set $S$, let $\operatorname{ch}(S)$ denote the characteristic function of $S$. Let $\delta_{N}$ be the modulus character on $M$ with respect to $N$. For $m \in M$, define functions $f^{\prime}[m] \in$ $\mathscr{H}_{K^{\circ}}(M)$ and $f[m] \in \mathscr{H}_{K}(G)$ by

$$
\begin{gathered}
f^{\prime}[m]=\operatorname{meas}\left(K^{0} m K^{0}\right)^{-1} \operatorname{ch}\left(K^{0} m K^{0}\right), \\
f[m]=\operatorname{meas}(K m K)^{-1} \delta_{N}^{-1 / 2}(m) \operatorname{ch}(K m K) .
\end{gathered}
$$

Let $(\pi, V)$ be an admissible $G^{*}$-module and let $\rho=r_{M G}(\pi)$. Let $W=V_{N}=$ $V / V(N)$ and let $T: V \rightarrow V_{N}=V / V(N)$ be the natural map. Note that $T$ commutes with the action of $\varepsilon$.

LeMma 7.2. Let $m \in M^{+}$and $w \in V^{K}$. Then $T(\pi(f[m]) w)=\rho\left(f^{\prime}[m]\right) T(w)$.

Proof. We have

$$
\operatorname{meas}(K m K)^{-1} \int_{K m K} \pi(x) w d x=\operatorname{meas}(K)^{-1} \int_{K} \pi(k) \pi(m) w d k .
$$

Since $m^{-1} K^{-} m \subset K^{-}, \pi(m) w$ is fixed by $K^{-}$. Using the decomposition $K=$ $K^{+} K^{0} K^{-}$, we obtain

$$
\operatorname{meas}\left(K^{+}\right)^{-1} \operatorname{meas}\left(K^{0}\right)^{-1} \int_{K^{+}} \int_{K^{0}} \pi(n) \pi(k) \pi(m) w d n d k
$$

and, modulo $V(N)$, this is equal to

$$
\operatorname{meas}\left(K^{0}\right)^{-1} \int_{K^{0}} \pi(k) \pi(m) w d k=\operatorname{meas}\left(K^{0} m K^{0}\right)^{-1} \int_{K^{0} m K^{0}} \pi(x) w d x .
$$

Fix $a \in Z_{M} \cap M^{+}$. It is easily verified (cf. $[3, \S 5.3]$ ) that for all $m \in M^{+}$, $f\left[a^{n} m\right]=f[a]^{n} f[m]$ for $n \geq 0$ and $f^{\prime}\left[a^{n} m\right]=f^{\prime}[a]^{n} f^{\prime}[m]$ for all $n$. By Proposition 3.3 of [6], for some large positive integer $l$, the subspace $U=\pi\left(f\left[a^{l}\right]\right) V^{K}$ of $V^{K}$ is stable under $\pi(f[a])$ and $T$ maps $U$ isomorphically to the space $W^{0}$ of $K^{0}$-fixed vectors in $W$. Hence $\pi\left(f\left[a^{l} m\right]\right) \pi(\varepsilon) V^{K} \subset U$ and since $T_{0} \pi(\varepsilon)=\rho(\varepsilon)_{0} T$, we have

(*) $\operatorname{Tr}\left(\pi\left(f\left[a^{l} m\right]\right) \pi(\varepsilon) \mid V^{K}\right)=\operatorname{Tr}\left(\pi\left(f\left[a^{l} m\right]\right) \pi(\varepsilon) \mid U\right)=\operatorname{Tr}\left(\rho\left(f^{\prime}\left[a^{l} m\right]\right) \rho(\varepsilon) \mid W^{0}\right)$

by Lemma 7.2 for all $m \in M^{+}$.

Let $\mathcal{M}$ be the subspace of functions $f$ in $\mathcal{H}_{K}(M)$ such that $r_{M G}^{*}\left(\chi_{f}\right) \in F_{\mathrm{tr}}(G)$. The equality (*) shows that $r_{M G}^{*}\left(\chi_{f^{\prime}\left[a^{l} m\right]}\right)=\chi_{f\left[a^{\prime} m\right]}$ and hence that $f^{\prime}\left[a^{l} m\right] \in \mathcal{M}$ for all $m \in M^{+}$. For every $m \in M, a^{N} m \in M^{+}$for some $N$ and therefore $f^{\prime}[a]^{N+l} f^{\prime}[m] \in J$. Since the functions $f^{\prime}[m] \operatorname{span} \mathcal{H}_{K^{0}}(M)$, the next lemma follows. 
LEMMA 7.3. Let $a \in M^{+} \cap Z_{M}$. Then for all $f \in \mathcal{H}_{K^{0}}(M), f^{\prime}[a]^{N} f \in \mathcal{M}$ for some positive integer $N$.

To prove Proposition 7.1, it must be shown that $\mathcal{M}=\mathcal{H}_{K^{0}}(M)$. We may argue as in [3]. As shown in [2], $\mathscr{H}_{K^{0}}(M)$ is a finitely-generated $Z(M)$-module, and, via the homomorphism $Z(G) \rightarrow Z(M)$, it becomes a finitely-generated $Z(G)$ module. Since $r_{L M}^{*}$ is a map of $Z(G)$-modules and $F_{\mathrm{tr}}(G)$ is a $Z(G)$-submodule of $R\left(M^{*}\right)^{\prime}, \mathcal{M}$ is a $Z(G)$-submodule of $\mathcal{H}_{K^{0}}(M)$. We deduce that for $a \in Z_{M} \cap M^{+}$, $f^{\prime}[a]^{N} \mathcal{H}_{K^{\circ}}(M)=\mathcal{M}$ for some $N$. Since $f^{\prime}[a]$ is invertible in $\mathcal{H}_{K^{0}}(M), \mathcal{H}_{K^{\circ}(M)}=\mathcal{M}$. This completes the proof of Proposition 7.1.

Let $\pi \in \operatorname{Irr}(G)^{\varepsilon}$. By [7], the distribution $f \rightarrow \operatorname{Tr}(\pi(f) \pi(\varepsilon))$ on $\mathcal{H}(G)$ is given by integration against a locally integrable function $\chi_{\pi \varepsilon}(\gamma)$ which is locally constant on the open subset of $\varepsilon$-regular elements in $G(\gamma \in G$ is called $\varepsilon$-regular if $\gamma \rtimes \varepsilon$ is "discriminant regular" in $G^{*}$ as defined in $\S 2.2$ of [7]). As was pointed out to me by L. Clozel, an analogue of Casselman's theorem holds in the twisted case. This is given in the next proposition.

For $g \in G^{*}$, let $P_{g}$ denote the parabolic subgroup associated to $g$. Recall that $P_{g}$ is defined as the set of $x \in G$ such that $\left\{\operatorname{ad}\left(g^{N}\right) x: N \in \mathbf{Z}^{+}\right\}$is bounded in $G$. Observe that $P_{x g x^{-1}}=x P_{g} x^{-1}$ and that $g\left(P_{g}\right) g^{-1}=P_{g}$.

Fix $g=\gamma \rtimes \varepsilon \in G^{*}$ where $\gamma \in G$. Let $y \in G$ be such that $y P_{g} y^{-1}$ is standard. Since $y^{-1} g y=y^{-1} \gamma \varepsilon(y) \rtimes \varepsilon$, we may and shall choose $\gamma$ within its $\varepsilon$-conjugacy class so that $P_{g}$ is standard. Set $P=P_{g}$. Then $g P g^{-1}=P$, that is, $\gamma \varepsilon(P) \gamma^{-1}=P$. But $\varepsilon(P)$ is also standard and conjugate to $P$, hence $\varepsilon(P)=P$ and $\gamma \in P$. Let $P=M N$ be the standard decomposition of $P$. If $\pi \in \operatorname{Irr}(G)^{\varepsilon}$, then $\gamma$ and $g$ act on the Jacquet module $\pi_{N}$ through the projection of $\gamma$ onto $M$ via the map $P \rightarrow P / N=M$.

Proposition 7.4. Let $(\pi, V) \in \operatorname{Irr}(G)^{\varepsilon}$. Let $\gamma \in G$ be an $\varepsilon$-regular element and set $g=\gamma \rtimes \varepsilon$. Assume that $\gamma$ is chosen within its $\varepsilon$-conjugacy class so that $P_{g}$ is standard. Let $P=P_{g}=M N$ and set $\rho=r_{M G}(\pi)$. Then $P$ is $\varepsilon$-invariant and $\chi_{\pi \varepsilon}(\gamma)=\delta_{N}(\gamma)^{1 / 2} \chi_{\rho \varepsilon}(\gamma)$.

PROOF. We may imitate the proof of [6]. Let $K$ be an $\varepsilon$-invariant open compact subgroup which is good with respect to $P=M N$ and is such that $\operatorname{ad}(g) K^{+} \subset K^{+}$, $\operatorname{ad}\left(g^{-1}\right) K^{-} \subset K^{-}$, and $\operatorname{ad}(g) K^{0}=K^{0}$. Let $g^{\prime}=\gamma^{\prime} \rtimes \varepsilon$, where $\gamma^{\prime}$ is the projection of $\gamma$ onto $M$. Let $f[g]$ and $f^{\prime}\left[g^{\prime}\right]$ be the normalized characteristic functions of $K g K$ and $K^{0} g^{\prime} K^{0}$, respectively, as before. We have that $f\left[g^{n}\right]=f[g]^{n}$ and $f^{\prime}\left[g^{\prime n}\right]=f\left[g^{\prime}\right]^{n}$ for $n \in \mathbf{Z}^{+}$. Let $W=V_{N}$. As in [6], there exists a positive integer $n_{0}$ such that for all $n \geq n_{0}$, the subspace $U=f\left[g^{n}\right] V^{K}$ is stable under $f[g]$ and maps injectively onto the subspace $W^{0}$ of $K^{0}$-fixed vectors in $W$. The relation

$$
\operatorname{Tr}\left(\pi\left(f\left[g^{n}\right]\right) \mid V^{K}\right)=\operatorname{Tr}\left(\pi\left(f\left[g^{n}\right]\right) \mid U\right)=\operatorname{Tr}\left(\rho\left(f^{\prime}\left[g^{\prime n}\right]\right) \mid W^{0}\right)
$$

holds for $n \gg 0$. Choose $K$ small enough so that $\chi_{\pi \varepsilon}$ is constant on $K m K$ and $\chi_{\rho \varepsilon}$ is constant on $K^{0} m K^{0}$. Then $\operatorname{Tr}\left(\pi(f[g]) \mid V^{K}\right)=\delta_{N}(\gamma)^{-1 / 2} \chi_{\pi} \varepsilon(\gamma)$ and $\operatorname{Tr}\left(\rho\left(f^{\prime}\left[g^{\prime}\right]\right) \mid W^{0}\right)=\chi_{\rho \varepsilon}(\gamma)$.

8. In this section, we formulate and prove the twisted analogues of the results of $\S 5.4$ of $[3]$.

Let $M, N \in \mathcal{L}(G)^{\varepsilon}$ and let $W(N, M)$ be the set of representatives for $W_{N} \backslash W / W_{M}$ of minimal length. The automorphism $\varepsilon$ acts on $W, W_{M}$, and $W_{N}$, and preserves 
the length function, since $\varepsilon$ preserves $P_{0}$. Hence $\varepsilon$ acts on $W(N, M)$. Let $W(N, M)^{\varepsilon}$ be the set of fixed points of $\varepsilon$ in $W(N, M)$ and let $\Omega_{1}, \ldots, \Omega_{k}$ be the $\varepsilon$-orbits in $W(N, M)$. All elements of an orbit have the same length. Assume that the $\Omega_{j}$ are ordered so that the length is decreasing in $j$.

Let $M, N \in \mathcal{L}(G)^{\varepsilon}$ and let $F=r_{N G} \circ i_{M G}: R\left(M^{*}\right) \rightarrow R\left(N^{*}\right)$. For $w \in$ $W(M, N)$, let $N(w)=N \cap w M w^{-1}$ and $M(w)=M \cap w^{-1} N w$. If $w \in W(N, M)^{\varepsilon}$, then $N(w) \in \mathcal{L}(N)^{\varepsilon}$ and $M(w) \in \mathcal{L}(M)^{\varepsilon}$ and that $\rho \rightarrow i_{N N(w)} \circ w \circ r_{M(w) M}(\rho)$ is a functor from $R\left(M^{*}\right)$ to $R\left(N^{*}\right)$.

LEMMA 8.1. For all $\rho \in R\left(M^{*}\right)$,

$$
F(\rho)=\sum_{w \in W(N, M)^{\epsilon}} i_{N N(w)} \circ w \circ r_{M(w) M}(\rho) \quad \text { modulo } R\left(M^{*}\right)^{0} .
$$

PROOF. If we forget the $\varepsilon$-action and regard $F$ as a functor from the Grothendieck group of $M$-modules to the Grothendieck group of $N$-modules, then, according to [4], we have

$$
F(\rho)=\sum_{w \in W(N, M)} i_{N N(w)} \circ w \circ r_{M(w) M}(\rho) .
$$

This is proved as follows. Let $w_{1}, w_{2}, \ldots, w_{p}$ be an ordering of the elements of $W(N, M)$ such that the length $\left(w_{i}\right) \geq \operatorname{length}\left(w_{i+1}\right)$. A functorial filtration $F_{1} \subset$ $F_{2} \subset \cdots \subset F_{p}=F$ of $F$ is defined so that

$$
F_{i}(\rho) / F_{i+1}(\rho)=i_{N N\left(w_{i}\right)} \circ w_{i} \circ r_{M\left(w_{i}\right) M}(\rho) .
$$

Let $P=M P_{0}=M U$ and $Q=N P_{0}=N U^{\prime}\left(U, U^{\prime}=\right.$ unipotent radicals of $P$ and $Q$, respectively). If $V$ is the space of $\rho$, then $i_{G M}(\rho)$ acts on the space of locally constant functions $\varphi: G \rightarrow V$ such that $\varphi(m n g)=\delta_{U}(m)^{1 / 2} \rho(m) \varphi(g)$. Let $W_{j}$ be the subspace of those $\varphi$ with support in $P w_{1} Q \cup P w_{2} Q \cup \cdots \cup P w_{j} Q$. Then $W_{j}$ is $Q$-invariant. By definition, $F_{i}(\rho)$ is the image of $W_{j}$ under $r_{N G}$.

Let $t_{i}=\left|\Omega_{i}\right|$. We may assume that $w_{j}$ are ordered so that the first $t_{1}$ elements belong to $\Omega_{1}$, the next $t_{2}$ elements belong to $\Omega_{2}$, etc. Define $F_{i}^{\prime}=F_{k(i)}$ where $k(i)=t_{1}+t_{2}+\cdots+t_{i}$. Then it is clear that $F_{i}^{\prime}(\rho)$ is invariant under $\varepsilon$ and hence is an $N^{*}$-module. Hence $F_{i}^{\prime}(\rho) / F_{i-1}^{\prime}(\rho)$ is an $N^{*}$-module and the restriction of $F_{i}^{\prime}(\rho) / F_{i-1}^{\prime}(\rho)$ to $N$ is a direct sum of $t_{i} N$-modules which are permuted by the action of $\varepsilon$. In particular, if $t_{i}>1$, then $F_{i}^{\prime}(\rho) / F_{i-1}^{\prime}(\rho)$ satisfies the hypothesis of Corollary 2.3 and hence belongs to $R\left(N^{*}\right)^{0}$.

For $M \in \mathcal{L}(G)^{\varepsilon}$, let $T_{M}=i_{G M} \circ r_{M G}: R\left(G^{*}\right) \rightarrow R\left(G^{*}\right)$.

LEMMA 8.2. Assume that $N, M \in \mathcal{L}(G)^{\varepsilon}$. Let $\rho \in R\left(M^{*}\right)$. Then

(a) $T_{N} \circ i_{G M}(\rho)=\sum_{w \in W(M, N)^{\varepsilon}} i_{G M(w)} \circ r_{M(w) M}(\rho)$ modulo $R\left(G^{*}\right)^{0}$.

(b) $T_{N} \circ T_{M}(\pi)=\sum_{w \in W(M, N)^{\varepsilon}} T_{M(w)}(\pi)$ modulo $R\left(G^{*}\right)^{0}$.

Proof. If $w \in W(M, N)^{\varepsilon}$, then $N(w)=w M(w) w^{-1}$. As follows from Lemma 5.4(iii) of [3], $i_{G N(w)} \circ w(\sigma)=i_{G M(w)}(\sigma)$ for all $\sigma \in R\left(M(w)^{*}\right)$. Part (a) follows 
from Lemma 8.1. For part (b), we have

$$
\begin{aligned}
r_{N G} \circ i_{G M} & \circ r_{M G}(\pi) \\
& =\sum_{w \in W(M, N)^{\varepsilon}} i_{N N(w)} \circ w \circ r_{M(w) M}\left(r_{M G}(\pi)\right) \text { modulo } R\left(M^{*}\right)^{0} \\
& =\sum_{w \in W(M, N)^{\varepsilon}} i_{N N(w)} \circ w \circ r_{M(w) G}(\pi) \text { modulo } R\left(M^{*}\right)^{0}
\end{aligned}
$$

and hence

$$
T_{N} \circ T_{M}(\pi)=\sum_{w \in W(M, N)^{\varepsilon}} i_{G N(w)} \circ w \circ r_{M(w) G}(\pi) \text { modulo } R\left(M^{*}\right)^{0}
$$

and (b) follows.

9. For $M \in \mathcal{L}(G)$, let $X(M)$ denote the group of unramified characters of $M$. Put $d(M)=\operatorname{dim}(X(M))$. For $m \in \mathcal{L}(G)^{\varepsilon}$, let $X(M)^{\varepsilon}$ be the set of fixed points of $\varepsilon$ in $X(M)$. Define a decreasing filtration $\left\{R^{i}\right\}$ of $R\left(G^{*}\right)$ by

$$
R^{i}=R\left(G^{*}\right)^{0}+\sum_{\substack{M \in \mathcal{L}(G)^{\epsilon} \\ d(M) \geq i}} i_{G M}\left(R\left(M^{*}\right)\right)
$$

Let $R\left(G^{*}\right)^{I}=R^{d(G)+1}$. An element $\pi \in \operatorname{Irr}(M)^{\varepsilon}$ will be called $\varepsilon$-discrete if it does not lie in $R\left(G^{*}\right)^{I}$.

Lemma 8.2(a) shows that $T_{N}$ preserves the filtration $\left\{R^{i}\right\}$ of $R\left(G^{*}\right)$ for $N \in$ $\mathcal{L}(G)^{\varepsilon}$. Let $P(N)=\operatorname{Card}\left(\left\{w \in W(N, N)^{\varepsilon}: w N w^{-1}=N\right\}\right)$. Then for $d=$ $d(N), M \in \mathcal{L}(G)^{\varepsilon}$, and $\sigma \in R\left(M^{*}\right):$

$$
T_{N}\left(i_{G M}(\sigma)\right)= \begin{cases}P(N) i_{G M}(\sigma) & \text { modulo } R^{d+1} \text { if } M \text { is conjugate to } N, \\ 0 & \text { modulo } R^{d+1} \text { if } M \text { is not conjugate to } N .\end{cases}
$$

Set $A_{d}=\prod\left(T_{N}-P(N)\right)$, the product over $N \in \mathcal{L}(G)^{\varepsilon}$ such that $d(N)=N$. Then $A_{d}$ preserves the filtration $\left\{R^{i}\right\}$ and maps $R^{d}$ to $R^{d+1}$. Hence the operator

$$
A=A_{d\left(M_{0}\right)} \circ A_{d\left(M_{0}\right)} \circ \cdots \circ A_{d(G)+1}
$$

maps $R^{I}$ to $R\left(G^{*}\right)^{0}$. By Lemma 8.2(b), there exist $C_{m} \in \mathbf{Q}$ and $P \in \mathbf{Z}$ such that

$$
A(\pi)=P\left(1-\sum c_{M} T_{M}\right)(\pi) \quad \text { modulo } R\left(G^{*}\right)^{0},
$$

where the sum is over $M \in \mathcal{L}(G)^{\varepsilon}$.

If $F \in R\left(G^{*}\right)^{\prime}$, we will call $F \varepsilon$-discrete if its vanishes on $R\left(G^{*}\right)^{I}$. Let $R\left(G^{*}\right)_{\mathrm{disc}}^{\prime}$ denote the subspace of $\varepsilon$-discrete functions in $R\left(G^{*}\right)^{\prime}$.

Proposition 9.1. Let $F \in R\left(G^{*}\right)^{\prime}$ and define

$$
F^{d}=F-\sum c_{M} r_{M G}^{*} i_{G M}^{*}(F) \text {. }
$$

Then $F^{d} \in R\left(G^{*}\right)_{\text {disc }}^{\prime}$.

Proof. By definition, $F^{d}(\pi)=P^{-1} F(A(\pi))$ for $\pi \in R\left(G^{*}\right)$. If $\pi \in R\left(G^{*}\right)^{I}$, then $A(\pi) \in R\left(G^{*}\right)^{0}$ and so $F(A(\pi))=0$. 
10. Define a map inf: $\operatorname{Irr}(G)^{\varepsilon} \rightarrow \Theta(G)$ by sending $\pi \in \operatorname{Irr}(G)^{\varepsilon}$ to a cuspidal pair $(M, \rho)$ such that $\pi$ is a constituent of $i_{G M}(\rho)$. The pair $(M, \rho)$ is well defined modulo conjugation by $W$ by the results of $[\mathbf{4}]$.

If $\inf (\pi)=\theta$ for some $\varepsilon$-discrete $\pi \in \operatorname{Irr}(G)^{\varepsilon}$, then $\theta$ will be called discrete. For $\Theta$ a component of $\Theta(G)$, let $\Theta_{\text {disc }}$ be the set of discrete $\theta$ in $\Theta$. For $M \in \mathcal{L}(G)^{\varepsilon}$, let $X(M)^{\varepsilon}$ be the subgroup of $X(M)$ fixed by $\varepsilon$.

The purpose of this section is to prove the following proposition.

Proposition 10.1. $\Theta_{\mathrm{disc}}$ is the union of finitely many $X(G)^{\varepsilon}$-orbits.

We first prove some preliminary results.

LEMMA 10.2. Let $\pi \in \operatorname{Irr}(G)^{\varepsilon}$ be an $\varepsilon$-discrete representation. Then there exists an $\varepsilon$-discrete tempered representation $\pi^{\prime} \in R(\theta)$ such that $\inf \left(\pi^{\prime}\right)$ is in the $X(G)^{\varepsilon}$-orbit of $\inf (\pi)$.

Proof. By the Langlands classification, to every $\pi \in \operatorname{Irr}(G)$ is associated Langlands data $(P, \sigma, \chi)$ consisting of a standard parabolic subgroup $P$, a tempered representation $\sigma$ of the Levi factor $M$ of $P$, and a character $\chi$ of $M$ which is positive with respect to the unipotent radical of $P$, such that $\pi$ is the unique irreducible quotient of $i_{G, M}(\chi \sigma)$. The triple $(P, \sigma, \chi)$ is unique. The Langlands data associated to $\varepsilon(\pi)$ is $(\varepsilon(P), \varepsilon(\sigma), \varepsilon(\chi))$. In particular, if $\pi \in \operatorname{Irr}(G)^{\varepsilon}$, then $(P, \sigma, \chi)$ is $\varepsilon$-invariant. Let $\rho$ be a supercuspidal representation of a Levi subgroup $M^{\prime}$ contained in $M$ such that $\chi \sigma$ is a constituent of $i_{M M^{\prime}}(\rho)$. Then each constituent of $i_{G M}(\chi \sigma)$ is also a constituent of $i_{G M^{\prime}}(\rho)$. We have

$$
\pi=i_{G M}(\chi \sigma)-\sum \pi_{i} \quad\left(\text { modulo } R\left(G^{*}\right)^{0}\right),
$$

where the $\pi_{i}$ are the irreducible $\varepsilon$-invariant constituents of $i_{G M}(\chi \sigma)$ distinct from $\pi$ (provided that the choice of the actions of $\varepsilon$ on $\pi$ and the $\pi_{i}$ are chosen compatible with that on $i_{G M}(\chi \sigma)$; we may assume this without loss of generality). Similarly, if $\left(P_{i}, \sigma_{i}, \chi_{i}\right)$ is $\left(\varepsilon\right.$-invariant) Langlands data for $\pi_{i}$, then $\pi_{i}=i_{G_{i} M_{i}}\left(\chi_{i} \sigma_{i}\right)-\sum \pi_{j i}$ (modulo $\left.R\left(G^{*}\right)^{0}\right)$. The process can be continued until we obtain an equality of the form

$$
\pi=\sum a_{j} i_{G_{j} M_{j}}\left(\chi_{j} \sigma_{j}\right)+\sum b_{k} \pi_{k} \quad\left(\operatorname{modulo} R\left(G^{*}\right)^{0}\right),
$$

where $a_{j}, b_{k} \in \mathbf{Z}, M_{j} \neq G$, and the $\pi_{k}$ are essentially tempered irreducible, $\varepsilon$ invariant representations (the process stops after finitely many steps by Lemma 2.13, p. 334 of [5]). The sum over $\pi_{k}$ is nonempty since $\pi$ is $\varepsilon$-discrete and there is at least one element $\pi_{m}$ in the sum which is $\varepsilon$-discrete. The $\pi_{k}$ are constituents of $i_{G M^{\prime}}(\rho)$ (Proposition 4.7 of [11]) and hence $\inf \left(\pi_{m}\right)=\inf (\pi)$. Let $\omega$ be the central character of $\pi_{k}$. Then the character $|\omega(z)|$ is $\varepsilon$-invariant and there exists an element $\chi \in X(G)^{\varepsilon}$ whose restriction to the center $Z$ of $G$ is $|\omega(z)|$. Hence $\pi_{m} \otimes \chi^{-1}$ is an $\varepsilon$-discrete tempered representation of $G$ and $\inf \left(\pi_{M} \otimes \chi\right)=\inf (\pi)$.

For $M \in \mathcal{L}(G)$, let $X(M)_{u}^{\varepsilon}$ be the subgroup of unitary characters in $X(M)^{\varepsilon}$.

Proposition 10.3. Let $M \in \mathcal{L}(G)$ and suppose that $s \varepsilon(M)=M$, where $s \in W$. Then there is a finite set $V$ of a square-integrable representations $\delta^{\prime}$ of $M$ such that $s \varepsilon\left(\delta^{\prime}\right)=\delta^{\prime}$, and a finite union $F$ of $X(G)_{u}^{\varepsilon}$-orbits of unitary characters of $M$ with the following property: if $\delta$ is square-integrable representation of $M$ such 
that $s \varepsilon(\delta)=\delta$ and $i_{G M}(\delta)$ contains an $\varepsilon$-discrete, $\varepsilon$-invariant representation $\pi$ such that $\inf (\pi) \in \Theta$, then $\delta=\delta^{\prime} \otimes \chi$ where $\delta^{\prime} \in V$ and $\chi \in F$.

ProOF. We begin with some lemmas. The first is taken from [9].

LEMMA 10.4. Let $M \in \mathcal{L}(G)$ and let $s \in W$ such that $s \varepsilon(M)=M$. Then there exists a Levi subgroup $L \in \mathcal{L}(G)^{\varepsilon}$ and an element $w \in W$ such that $L \supset w^{-1} M w$, $s \varepsilon(w) \in L$, and $w^{-1}\left(\mathcal{A}(M)^{s \varepsilon}\right)=\mathcal{A}(L)^{\varepsilon}$.

PROOF. We use induction on the semisimple rank of $G$. If $A(M)^{s \varepsilon}=A(G)^{\varepsilon}$, the assertion is clear. Otherwise, there is an element $\chi \in A(M)^{s \varepsilon}$ on which all roots do not vanish. This defines a proper parabolic subgroup $P_{1}$ containing $M$ which is fixed by $s \varepsilon$. Choose $w \in W$ so that $P^{\prime \prime}=w^{-1} P_{1} w$ is a standard parabolic subgroup and set $M^{\prime}=w^{-1} M w$. Let $s^{\prime}=w^{-1} s \varepsilon(w)$. Then $s^{\prime} \varepsilon\left(M^{\prime}\right) s^{\prime-1}=M^{\prime}$ and $\varepsilon\left(P^{\prime \prime}\right)=s^{\prime-1} P^{\prime \prime} s^{\prime}$. Since $P^{\prime \prime}$ is standard, this implies that $\varepsilon\left(P^{\prime \prime}\right)=P^{\prime \prime}$ and that $s^{\prime} \in W_{M^{\prime \prime}}$, where $M^{\prime \prime}$ is the Levi factor of $P^{\prime \prime}$. The induction assumption can be applied to $M^{\prime \prime}$ with $M^{\prime}$ in place of $M$.

Let $M, L \in \mathcal{L}(G)$ and suppose that $M \subset L$. Let $\rho$ be an $M$-module. Since $i_{G M}=$ $i_{G L} \circ i_{L M}$ and $i_{G L}$ is right adjoint to $r_{L G}$, there is a canonical map $r_{L G} \circ i_{G M}(\rho) \rightarrow$ $i_{L M}(\rho)$ which corresponds to the identity in $\operatorname{Hom}\left(i_{G M}(\rho), i_{G M}(\rho)\right)$. Explicitly, the map is obtained as follows. The representation $i_{G M}(\rho)\left(\right.$ resp. $\left.i_{L M}(\rho)\right)$ acts on a space of functions on $G$ (resp. $L$ ). Regarding $i_{G M}(\rho)$ as an $L$-module, there is a surjective map of $L$-modules $i_{G M}(\rho) \rightarrow i_{L M}(\rho)$ given by restricting a function $f$ on $G$ to $L$. This map factors to give the canonical map $r_{L G} \circ i_{G M}(\rho) \rightarrow i_{L M}(\rho)$ which is also surjective. From this we also obtain a canonical map $c: r_{M G} \circ i_{G M}(\rho) \rightarrow$ $r_{M L} \circ i_{L M}(\rho)$.

Let $\tau$ be another $M$-module and let $T^{\prime}: i_{L M}(\rho) \rightarrow i_{L M}(\tau)$. By adjunction, we have a map $r_{L G} \circ i_{G L}\left(T^{\prime}\right): r_{L G} \circ i_{G L} \circ i_{L M}(\rho) \rightarrow i_{L M}(\tau)$ from which it follows that the map $r_{M G} \circ i_{G L}\left(T^{\prime}\right): r_{M G} \circ i_{G M}(\rho) \rightarrow \tau$ factors as

$$
r_{M G} \circ i_{G M}(\rho) \stackrel{c}{\rightarrow} r_{M L} \circ i_{L M}(\rho) \stackrel{r_{M L}\left(T^{\prime}\right)}{\longrightarrow} \tau .
$$

In particular, a map $T: i_{G M}(\rho) \rightarrow i_{G M}(\tau)$ is induced from a map $T^{\prime}: i_{L M}(\rho) \rightarrow$ $i_{L M}(\tau)$ if and only if $r_{M G}(T)$ factors through $c$.

Let $w_{1}, \ldots, w_{t}$ be an ordering of the set of representatives of $W_{M} \backslash W / W_{M}$ of minimal length such that length $\left(w_{i}\right) \geq \operatorname{length}\left(w_{i+1}\right)$. Let $P$ be the standard parabolic subgroup with Levi factor $M$ and let $W_{j}(\rho)$ be the subspace of $i_{G M}(\rho)$ consisting of functions supported in $P w_{1} P \cup P w_{2} P \cup \cdots \cup P w_{j} P$. Let $F_{j}(\rho)$ be the image of $W_{j}(\rho)$ in $r_{M G} \circ i_{G M}(\rho)$. As in $\S 8, F_{j}(\rho) / F_{j-1}(\rho)$ is isomorphic to $i_{M M\left(w_{j}\right)} \circ w_{j} \circ r_{M\left(w_{j}^{-1}\right) M}(\rho)$ where we set $M(w)=w M w^{-1} \cap M$. Observe that if the double coset $W_{M} w_{j} W_{M}$ is contained in $W_{L}$, then $w_{j} \in W_{L}$.

LEMMA 10.5. Let $\rho, \tau$ be irreducible representations of $M$ and let $T: i_{G M}(\rho) \rightarrow$ $i_{G M}(\tau)$ be a map of $G$-modules. Let $r_{M G}(T)$ be the associated map from $r_{M G} \circ$ $i_{M G}(\rho)$ to $\tau$. Suppose that for some $j, F_{j-1}(\rho) \in \operatorname{Ker}\left(r_{M G}(T)\right)$ and that $r_{M G}(T)$ induces a surjective map $F_{j}(\rho) / F_{j-1}(\rho) \rightarrow \tau$. Suppose that $w_{j} \in W_{L}$. Then $T=$ $i_{G L}\left(T^{\prime}\right)$ where $T^{\prime}: i_{L M}(\rho) \rightarrow i_{L M}(\tau)$.

PROOF. By the above discussion, we must show that $r_{M G}(T)$ factors through the canonical map $c: r_{M G} \circ i_{G M}(\rho) \rightarrow r_{M L} \circ i_{L M}(\rho)$. Let $P^{\prime}$ be the standard parabolic 
subgroup of $L$ whose Levi factor is $M$. If $w_{j} \in W_{L}$, let $Z_{j}(\rho)$ be the subspace of $i_{L M}(\rho)$ consisting of functions supported in the union of the double cosets $P^{\prime} w_{i} P^{\prime}$, where $i$ ranges over the positive integers such that $i \leq j$ and $w_{i} \in W_{L}$ and let $G_{j}(\rho)$ be the image of $Z_{j}(\rho)$ in $r_{M L} \circ i_{L M}(\rho)$. If $j^{\prime}$ is the largest integer such that $j^{\prime}<j$ and $w_{j} \in W_{L}$, then $G_{j}(\rho) / G_{j^{\prime}}(\rho)$ is isomorphic to $i_{M M\left(w_{j}\right)} \circ w_{j} \circ r_{M\left(w_{j}^{-1}\right) M}(\rho)$.

Let $N^{\prime}$ be the unipotent radical of the standard parabolic subgroup of $G$ whose unipotent radical is $L$. Then $P=P^{\prime} N^{\prime}$ and hence if $w \in W_{L}, P w P=P^{\prime} w P$. It follows that $P w P \cap L^{\prime}=P^{\prime} w P^{\prime}$. On the other hand, if $w \in W-W_{L}$, then $P w P \cap L$ is empty since the Bruhat decompositions are disjoint. It follows that the $L$-module map $i_{G M}(\rho) \rightarrow i_{L M}(\rho)$ sends $W_{j}(\rho)$ onto $Z_{j}(\rho)$ and that $c$ maps $F_{j}(\rho)$ onto $G_{j}(\rho)$, inducing an isomorphism of $F_{j}(\rho) / F_{j-1}(\rho)$ onto $G_{j}(\rho) / G_{j^{\prime}}(\rho)$.

Let $K=\operatorname{Ker}\left(r_{M G}(T)\right)$. Then $r_{M G} \circ i_{G M}(\rho)=K+F_{j}(\rho)$ and $r_{M L} \circ i_{L M}(\rho)=$ $c(K)+G_{j}(\rho)$ since $c$ is surjective. If $x=k+g$, where $k \in c(K)$ and $g \in G_{j}(\rho)$, define $S(x)=T(f)$ where $f \in F_{j}(\rho)$ is any element such that $c(f)=g$. Then $S(x)$ is well defined since $T\left(F_{j-1}(\rho)\right)=0$ and $r_{M G}(T)=S \circ c$.

We now prove Proposition 10.3, basing our argument on that given in the proof of Lemma 4 of [8]. For the convenience of the reader, we reproduce those parts of [loc. cit] needed.

LEMMA 10.6. There are only finitely-many $X(M)_{u}$-orbits of square-integrable $\delta$ such that $i_{G M}(\delta)$ has a constituent $\pi$ with $\inf (\pi) \in \Theta$.

PROOF. There exists an open compact subgroup $K$ of $G$ such that the space $\pi^{K}$ of $K$-invariants is nonzero for all $\pi \in \operatorname{Irr}(G) \operatorname{such}$ that $\inf (\pi) \in \Theta$ [2]. If $\delta$ is a unitary square-integrable representation of $M$ such that $i_{G M}(\delta)$ has a constituent $\pi$ with $\inf (\pi) \in \Theta$, then $\delta^{K \cap M} \neq\{0\}$ by Frobenius reciprocity. The lemma follows if we show that the set of unitary square-integrable $\delta$ such that $\delta^{K \cap M} \neq\{0\}$ is finite modulo $X(M)_{u}$. Let $Z_{M}$ be the center of $M$. Since the restriction map from $X(M)_{u}$ to $\operatorname{Hom}_{u}\left(Z_{M}, \mathbf{C}^{*}\right)$ is finite, it will suffice to show that there are only finitely many such $\delta$ with a fixed central character $\chi$. By a theorem of HarishChandra (cf. [8]), the space ${ }^{0} C_{\chi}(M, K \cap M)$ of bi- $(K \cap M)$-invariant functions in the space of cuspidal Schwartz functions on $M$ which transform by $\chi$ under the $Z_{M}$ is finite-dimensional. Furthermore, ${ }^{0} C_{\chi}(M, K \cap M)$ contains the bi- $(K \cap M)$ invariant matrix coefficients of the square-integrable $\delta$ which transform under $Z_{M}$ by $\chi$. The finiteness follows.

Let $\delta$ be a unitary square-integrable representation of $M$ such that $s \varepsilon(\delta)=\delta$ and $i_{G M}(\delta)$ has an $\varepsilon$-discrete, $\varepsilon$-invariant constituent $\pi$ such that $\inf (\pi) \in \Theta$. Let $W\left(G, A_{M}\right)=N_{G}\left(A_{M}\right) / M$, where $N_{G}\left(A_{M}\right)$ is the normalizer of $A_{M}$ in $G$ and set $W_{\delta}=\left\{w \in W\left(G, A_{M}\right): \delta=w(\delta)\right\}$. Since there are only finitely many possibilities for $W_{\delta}$, it will suffice to prove the finiteness of the number of $X(G)_{u}^{\varepsilon}$-orbits in the set $F(\delta)$ of $\chi \in X(M)_{u}$ such that $s \varepsilon(\chi \delta)=\chi \delta, i_{G M}(\chi \delta)$ has an $\varepsilon$-discrete, $\varepsilon$-invariant constituent $\pi$ such that $\inf (\pi) \in \Theta$ and $W_{\delta \chi}=W_{\delta}$.

Let $\left\{\alpha_{1}, \ldots, \alpha_{k}\right\}$ be a basis for the rational co-characters of $A_{M}$ and let $\omega$ be a prime element in $F$. Let $C_{M}$ be the free abelian group generated by $\left\{\alpha_{j}(\omega)\right\}$. Then the center of $M / C_{M}$ is compact. If $\tau$ is an irreducible representation of $M$, let $\psi_{\tau}$ denote the character of $C_{M}$ by which $C_{M}$ acts on $\tau$. Let $H: M \rightarrow A(M)_{\mathbf{R}}$ denote the standard map, where $A(M)_{\mathbf{R}}=\operatorname{Hom}\left(X\left(A_{M}\right), \mathbf{Z}\right) \otimes \mathbf{R}$. The image of $C_{M}$ under $H$ is a lattice $\mathcal{L}$ in $A(M)_{\mathbf{R}}$. We identify $\operatorname{Hom}\left(C_{M}, \mathbf{C}^{*}\right)$ with $A(M)^{*} / \mathcal{L}$, 
where $\mathcal{A}(M)^{*}=X\left(A_{M}\right) \otimes \mathbf{C}$ and $\mathcal{L}^{\wedge}=\left\{\lambda \in \mathcal{A}(M)^{*}: \lambda(\mathcal{L}) \in(2 \pi i / \log (q)) \mathbf{Z}\right\}$, by associating to $\lambda \in \mathcal{A}(M)^{*}$ the character $c \rightarrow q^{\langle H(c), \lambda\rangle}$. Here $q$ is the cardinality of the residue field of $F$.

Suppose that $F(\delta)$ is infinite modulo the action of $X(G)_{u}^{\varepsilon}$. There are only finitely many characters $\chi \in X(M)_{u}$ whose restriction of $C_{M}$ is trivial and hence the set $\left\{\psi_{\chi \delta}: \chi \in F(\delta)\right\}$ is infinite modulo $X(G)_{u}^{\varepsilon}$. Observe that $W_{\delta}$ is stable under the action of $s \varepsilon$ since $s \varepsilon(w) s^{-1}(\delta)=\delta$ if $w \in W_{\delta}$. Let $W^{\prime}$ be the semidirect product of $W_{\delta}$ with the cyclic group generated by $s \varepsilon$. Let $\operatorname{Hom}_{u}\left(C_{M}, \mathbf{C}^{*}\right)$ be the group of unitary characters of $C_{M}$. Then $W^{\prime}$ acts on $\operatorname{Hom}_{u}\left(C_{M}, \mathbf{C}^{*}\right)$ and $\psi_{\chi \delta}$ is fixed by $W^{\prime}$ for all $\chi \in F(\delta)$. It follows that there are infinitely many $W^{\prime}$-fixed points in the torus $A(M)^{*} / \mathcal{L}^{-}$which are not in the image of $A(G)^{*}$. Since the action of $W^{\prime}$ on $A(M)^{*} / \mathcal{L}^{\wedge}$ is algebraic, there exists a nonzero vector $\lambda \in A(M)^{*}-\mathcal{A}(G)^{*}$ fixed by $W^{\prime}$. The action of $W^{\prime}$ on $A(M)^{*}=X\left(A_{M}\right) \otimes \mathbf{C}$ comes from an action of $W^{\prime}$ on $X(M)$ and hence $W^{\prime}$ fixes a sublattice $V$ of $X(M)$ which is not contained in $X(G)$. It follows that $W^{\prime}$ fixes a subtorus $A$ of $A_{M}$ which strictly contains $A_{G}$. According to [6, Lemma 1.1], the centralizer of $A$ is a Levi subgroup $L^{\prime}$ of $G$ which contains $M$. By construction, $W\left(L^{\prime}, A_{M}\right)$ contains $W_{\delta}$ and $L^{\prime}$ is stable under $s \varepsilon$. By Lemma 10.4, we may conjugate the entire situation and assume that there exists a proper $\varepsilon$-stable Levi factor $L$ which contains $L$ such that $s \in W_{L}$.

According to the Corollary to Theorem 2 of $[8]$, for $\chi \in F(\delta)$, every irreducible constituent of $i_{G M}(\delta \chi)$ is of the form $i_{G L}(\rho)$, where $\rho$ is an irreducible representation of $i_{L M}(\delta \chi)$. By hypothesis, $i_{G M}(\delta \chi)$ contains at least one $\varepsilon$-invariant, $\varepsilon$-discrete subrepresentation $\pi(\chi)$. Let $\rho(\chi)$ be the subrepresentation of $i_{L M}(\delta \chi)$ such that $i_{G L}(\rho(\chi))=\pi(\chi)$.

Since $L$ is $\varepsilon$-invariant and $s \in W_{L}$, the data $(M, \delta \chi)$ is conjugate to $(\varepsilon(M), \varepsilon(\delta \chi))$ in $L$. The set of subrepresentations of $i_{L M}(\delta \chi)$ is therefore stable under $\varepsilon$ and so $\varepsilon(\rho(\chi))$ is also a subrepresentation of $\left.i_{L M}(\delta \chi)\right)$. Since $\pi(\chi)$ is $\varepsilon$-discrete, $\rho(\chi)$ cannot be $\varepsilon$-invariant, but since $\pi(\chi)$ is $\varepsilon$-invariant, $i_{G L}(\rho(\chi))$ is isomorphic to $i_{G L}(\varepsilon(\rho(\chi)))$. Identify $\varepsilon(\rho(\chi))$ with a subrepresentation of $\left.i_{L M}(\delta \chi)\right)$ and let $\varphi(\chi)$ : $i_{G M}(\delta \chi) \rightarrow i_{G M}(\delta \chi)$ be any map which sends the summand $i_{G L}(\rho(\chi))=\pi(\chi)$ to the summand $i_{G L}(\varepsilon(\rho(\chi)))$. Then $\varphi(\chi)$ is not the identity map, it corresponds, by adjunction, to a map $\xi(\chi): r_{G M} \circ i_{G M}(\delta \chi) \rightarrow \delta \chi$ which is distinct from the standard map which corresponds by adjunction to the identity.

Let $F=\{\chi\}$ be a set of representatives for the $X(G)_{u}^{\varepsilon}$-orbits in $F(\delta)$. Since $F$ is infinite, there exists a nontrivial element $w \in W$ giving a shortest representative for a double coset in $W_{M} \backslash W / W_{M}$ such that $\xi(\chi)$ induces a map $i_{M M(w)} \circ$ $w \circ r_{M\left(w^{-1}\right) M}(\delta \chi) \rightarrow \delta \chi\left(\right.$ where $i_{M M(w)} \circ w \circ r_{M\left(w^{-1}\right) M}(\delta \chi)$ is regarded as a subquotient of $\left.r_{G M} \circ i_{G M}(\delta \chi)\right)$ for infinitely many $\chi \in F$. The representation $i_{M M(w)} \circ w \circ r_{M\left(w^{-1}\right) M}(\delta \chi)$ is isomorphic to $w(\chi) i_{M M(w)} \circ w \circ r_{M\left(w^{-1}\right) M}(\delta \chi)$ and hence $\xi(\chi)$ induces a map from $\chi^{-1} w(\chi) i_{M M(w)} \circ w \circ r_{M\left(w^{-1}\right) M}(\delta)$ to $\delta$. It follows that there are infinitely many $\chi \in F$ such that $\psi_{\chi \delta}$ is fixed by $w$ and by $W^{\prime}$. Let $W^{\prime \prime}$ be the group of automorphisms of $\operatorname{Hom}_{u}\left(C_{M}, \mathbf{C}^{*}\right)$ generated by $w$ and $W^{\prime}$. We may repeat the above argument to obtain a proper $\varepsilon$-invariant Levi subgroup $L^{\prime}$ containing $M$ such that $W_{\delta} \in W\left(L^{\prime}, A_{M}\right)$ and $s, w \in W_{M^{\prime}}$. By Lemma 10.5, the map $\varphi(\chi)$ is obtained by induction from a map $\varphi_{0}(\chi): i_{L^{\prime} M}(\delta \chi) \rightarrow i_{L^{\prime} M}(\delta \chi)$. There are irreducible subrepresentations $\tau(\chi), \tau^{\prime}(\chi)$ of $i_{L^{\prime} M}(\delta \chi)$ such that $\varepsilon(\tau(\chi))$ is isomorphic to $\tau^{\prime}(\chi), i_{G L^{\prime}}(\tau(\chi))=i_{G L}(\rho(\chi))$, and $i_{G L^{\prime}}\left(\tau^{\prime}(\chi)\right)=i_{G L}(\varepsilon(\rho(\chi)))$. The 
map $\varphi_{0}(\chi)$ must take $\tau(\chi)$ to $\tau^{\prime}(\chi)$ and hence $\tau(\chi)$ is $\varepsilon$-invariant. This contradicts the assumption that $\pi(\chi)$ is $\varepsilon$-discrete and proves the proposition.

We now prove Proposition 10.1. Let $\theta \in \Theta_{\text {disc }}$. By Lemma 10.2, we may assume, modulo the action of $X(G)^{\varepsilon}$, that $\inf (\pi)=\theta$ for some $\varepsilon$-discrete tempered representation $\pi$. There exists a standard parabolic subgroup $P=M N$ and a unitary square-integrable representation $\delta$ of $M$ such that $\pi$ is a subrepresentation of $i_{G M}(\delta)$. The data $(M, \delta)$ is determined up to conjugacy by $\pi$ and since $\varepsilon(\pi)$ is a subrepresentation of $i_{G \varepsilon(M)}(\varepsilon(\delta))$, there exists an element $s \in W$ such that $s \varepsilon(M)=M$ and $s \varepsilon(\delta)=\delta$. By Proposition 10.3, there are only finitely many pairs $(M, \delta)$ such that $i_{G M}(\delta)$ contains an $\varepsilon$-invariant, $\varepsilon$-discrete subrepresentation, modulo the action of $X(G)_{u}^{\varepsilon}$. Proposition 10.1 follows

11. Let $\varepsilon$ act on $\Theta(G)$ by sending $(M, \rho)$ to $(\varepsilon(M), \varepsilon(\rho))$. Then $\varepsilon$ permutes the set of components of $\Theta(G)$. Let $\pi \in \operatorname{Irr}(G)^{\varepsilon}$ and suppose that $\pi$ is a constituent of $i_{G M}(\rho)$ where $(M, \rho)$ is a cuspidal pair. Then $\pi$ is a constituent of both $i_{G M}(\rho)$ and $\varepsilon\left(i_{G M}(\rho)\right)=i_{G \varepsilon(M)}(\varepsilon(\rho))$. It follows that $\varepsilon(\inf (\pi))=\inf (\pi)$, that is, there exists an element $s \in W$ such that $M=s \varepsilon(M) s^{-1}$ and $s \varepsilon(\rho)=\rho$. If $\Theta$ is a component of $\Theta(G)$ such that $\inf (\pi) \in \Theta$ for some $\pi \in \operatorname{Irr}(G)^{\varepsilon}$, then $\varepsilon(\Theta)=\Theta$.

For $\Theta$ such that $\varepsilon(\Theta)=\Theta$, let $R(\Theta)$ be the subspace of $R\left(G^{*}\right)$ generated by $\{\pi \in$ $\left.\operatorname{Irr}(G)^{\varepsilon}: \inf (\pi) \in \operatorname{Irr}(G)^{\varepsilon}\right\}$. In order to state the twisted Paley-Wiener theorem (Theorem 11.2 below), define the subspace $F_{g}(G)$ of elements $F \in R\left(G^{*}\right)^{\prime}$ such that:

(I) There is a finite set $S$ of $\varepsilon$-stable components of $\Theta(G)$ such that $F$ is supported in $\sum_{\Theta \in S} R(\Theta)$.

(II) For every $L \in \mathcal{L}(G)^{\varepsilon}$ and $\rho \in \operatorname{Irr}(L)^{\varepsilon}$, the function $\psi \rightarrow F\left(i_{G L}(\psi \rho)\right)$ is a regular function on $X(L)^{\varepsilon}$.

For $M \in \mathcal{L}(G)^{\varepsilon}, F_{g}(M)$ is defined similarly.

LEMMA 11.1. (a) $F_{\mathrm{tr}}(G) \subset F_{g}(G)$.

(b) For all $M \in \mathcal{L}(G)^{\varepsilon}, i_{G M}^{*}\left(F_{g}(G)\right) \subset F_{g}(M)$.

ProOF. Let $K$ be an open compact subgroup of $G$. By [2], there are only finitely-many components $\Theta$ of $\Theta(G)$ such that $\pi^{k} \neq\{0\}$ for some $\pi \in \operatorname{Irr}(G)$ such that $\inf (\pi) \in \Theta$. Hence $\chi_{f}$ satisfies (I) for all $f \in \mathcal{H}_{K}(G)$. Let $L \in \mathcal{L}(G)^{\varepsilon}$ and $\rho \in \operatorname{Irr}(L)^{\varepsilon}$. The representations $i_{G L}(\psi \rho)$ for $\chi \in X(L)^{\varepsilon}$ all act on a fixed vector space $V$. Both the action of $\varepsilon$ and the subspace $V^{K}$ of $K$-fixed vectors in $V$ is independent of $\chi$. Furthermore, if we choose a basis for $V^{K}$, then $f$ acts by a matrix whose coefficients are polynomials on $X(L)^{\varepsilon}$, and hence $\chi_{f}$ satisfies (II). This proves (a). To prove (b), observe that if $F \in F_{g}(G)$, then $i_{G M}^{*}(F)$ satisfies (II) by transitivity of induction and $i_{G M}^{*}(F)$ satisfies (I) because the canonical map from $\Theta(M)$ to $\Theta(G)$ is finite.

PROPOSITION 11.2. $F_{\mathrm{tr}}(G)=F_{g}(G)$.

Proof. We follow [3]. Let $F \in F_{g}(G)$. For $\Theta$ an $\varepsilon$-stable component, let $1(\Theta) \in Z(G)$ be the characteristic function of $\Theta$. Then $F=\sum 1 \mid(\Theta) F$, where all but finitely many terms in the sum are zero, and $1(\Theta) F \in F_{g}(G)$. To prove that $F \in F_{\mathrm{tr}}(G)$, we may therefore assume that $F$ is supported in a single $\varepsilon$-stable component $\Theta$. We may further assume by induction that the theorem holds for all proper standard Levi subgroups of $G$. 
Let $F_{\text {disc }}(G)$ be the space of discrete functions in $F_{g}(G)$. Let $R\left(\Theta_{\text {disc }}\right)$ be the subspace of $R(\Theta)$ generated by the set of $\pi \in \operatorname{Irr}\left(G^{*}\right)$ such that $\inf (\pi) \in \Theta$ and $\inf (\pi)=\inf \left(\pi^{\prime}\right)$ for some $\varepsilon$-discrete $\pi^{\prime} \in \operatorname{Irr}(G)^{\varepsilon}$. Proposition 10.1 implies that $R\left(\Theta_{\text {disc }}\right)$ is generated by finitely many $X(G)^{\varepsilon}$-orbits of $G^{*}$-modules and hence for all $F \in F_{\text {disc }}(G)$, there exists $F^{\prime} \in F_{\mathrm{tr}}(G)$ such that $F-F^{\prime}$ vanishes on $R\left(\Theta_{\text {disc }}\right)$. (If $X(G)^{\varepsilon}=\{1\}$, the implication follows from the linear independence of twisted characters on $\mathcal{H}(G)$; in the general case we may argue as in $\S 4$ of $[3])$.

Suppose that $F \in F_{g}(G)$ and that $F$ vanishes on $R\left(\Theta_{\mathrm{disc}}\right)$. By Proposition 7.1 and the induction hypothesis, $r_{M G}^{*}\left(i_{G M}^{*}\left(F_{g}(G)\right)\right) \subset F_{\mathrm{tr}}(G)$ for $M \in \mathcal{L}(G)^{\varepsilon}$. Proposition 9.1 then implies that $F-F^{d} \in F_{\mathrm{tr}}(G)$, where $F^{d}$ is as defined in Proposition 9.1. However, $F^{d}=0$ if $F$ vanishes on $R\left(\Theta_{\text {disc }}\right)$ because the operators $i_{G M} \circ r_{M G}$ preserve $R\left(\Theta_{\text {disc }}\right)$.

\section{REFERENCES}

1. J. Arthur and L. Clozel, Base change for $\mathrm{GL}(n)$, preprint.

2. J. Bernstein and P. Deligne, Le "centre" de Bernstein, Représentations des Groupes Reductifs sur un Corps Local, Hermann, Paris, 1985.

3. J. Bernstein, P. Deligne and D. Kazhdan, Trace Paley-Wiener theorem for reductive p-adic groups, J. Analyse 47 (1986), 180-192.

4. J. Bernstein and A. Zelevinsky, Induced representations of reductive p-adic groups, Ann. Sci. Ecole Norm. Sup. (4) 10 (1977), 441-472.

5. A. Borel and N. Wallach, Continuous cohomology, discrete subgroups, and representations of reductive groups, Ann. of Math. Studies, no. 94, Princeton Univ. Press, Princeton, N. J., 1980.

6. W. Casselman, Characters and Jacquet modules, Math. Ann. 230 (1977), 101-105.

7. L. Clozel, Characters of non-connected reductive $p$-adic groups, preprint.

8. __ Sur une conjecture de Howe. I, Compositio Math. 56 (1985), 87-110.

9. Seminar on the trace formula, Institute for Advanced Study, Princeton, N. J., 1984.

10. J. Rogawski, Automorphic representations of unitary group in three variables, preprint.

11. __ Representations of $\mathrm{GL}(n)$ and division algebras over a p-adic field, Duke Math. J. 50 (1983), 161-196.

Department of Mathematics, University of California, Los Angeles, CaliFORNIA 90024 